\title{
Secondary electron emission from meteoric smoke particles inside the polar ionosphere
}

\author{
Carsten Baumann $^{1}$, Markus Rapp ${ }^{1,2}$, and Antti Kero ${ }^{3}$ \\ ${ }^{1}$ Deutsches Zentrum für Luft- und Raumfahrt, Institut für Physik der Atmosphäre, Oberpfaffenhofen, Germany \\ ${ }^{2}$ Meteorologisches Institut München, Ludwig-Maximilians-Universität München, Munich, Germany \\ ${ }^{3}$ Sodankylä Geophysical Observatory, University of Oulu, Tähteläntie 62, 99600, Sodankylä, Finland \\ Correspondence to: Carsten Baumann (carsten.baumann@dlr.de)
}

Received: 24 April 2016 - Accepted: 31 May 2016 - Published: 23 June 2016

\begin{abstract}
The charging by secondary electron emission (SEE) from particles is known as a significant charging process in astrophysical plasmas. This work aims at evaluating the significance of SEE for charging of meteoric smoke particles (MSPs) in the Earth's polar atmosphere. Here, the atmosphere is subject to a bombardment of energetic electrons from the magnetosphere (and partly the sun). We employ the SEE formalism to MSPs in the upper mesosphere using electron precipitation fluxes for three different precipitation strengths. In addition, we address the possible effect of tertiary electron emission (TEE) from MSPs induced by atmospheric secondary electrons for one precipitation case. The SEE and TEE rates from MSPs of different sizes are compared to plasma attachment and photodetachment and photoionization rates of MSPs. The needed concentration of electrons and ions have been modeled with the Sodankylä Ion and Neutral Chemistry (SIC) model with included electron precipitation spectra as an additional ionization source. We find that secondary electron emission from MSPs is not a relevant charging mechanism for MSPs. The electron attachment to MSPs and photodetachment of negatively charged MSPs are the most important processes also during energetic electron precipitation.
\end{abstract}

Keywords. Ionosphere (ionosphere-atmosphere interactions)

\section{Introduction}

Secondary electron emission (SEE) occurs when an energetic electron hits and enters a material. Consequently, a secondary electron may be excited with enough energy to leave the ma- terial (Austin and Starke, 1902). This physical effect has been studied in various fields, e.g., material sciences (e.g., Bruining, 1954, and references therein), astrophysics (e.g., Goertz, 1989; Walch et al., 1995; Abbas et al., 2012) and space applications (e.g., Katz et al., 1986; Balcon et al., 2012). Also the Earth's atmosphere is subject to energetic electron precipitation, which is the reason for the well-studied phenomenon of the aurora (e.g., Rees, 1969). Furthermore, Anderson and Koons (1996) reported that SEE also has effects on the charge state of satellites when they orbit low over the poles within the auroral regions. There have also been studies covering SEE from atmospheric molecules in the context of auroral excitation mechanisms (e.g., Rees et al., 1969).

This work, however, aims at investigating whether SEE is also a relevant charging process for meteoric smoke particles (MSPs). Furthermore, MSPs are present in the mesosphere which coincides with the altitude region where much of the energy of the precipitating electrons is deposited and therefore are likely to be affected by SEE. These MSPs are particles, which originate from extraterrestrial matter that is injected into the atmosphere by evaporating meteors (Rosinski and Snow, 1961; Megner et al., 2006). The existence of these MSPs was proven by in situ measurements on sounding rockets (e.g., Havnes et al., 1996; Rapp et al., 2012), by spectrometers on board satellites (Hervig et al., 2009) and by means of incoherent-scatter radars (Strelnikova et al., 2007). MSPs have effects on the nucleation of ice particles in the mesosphere (e.g., Wilms et al., 2016, and references therein) and influences on the ionospheric charge balance (Friedrich et al., 2012; Baumann et al., 2013; Plane et al., 2014; Asmus et al., 2015) and ion chemistry (Baumann et al., 2015) of the D region. 
This study of the SEE from MSPs in the polar atmosphere investigates its relevance by comparing the rates of SEE with other MSP-related charging rates. The paper is structured as follows: Sect. 2 covers the formalism to derive SEE rates. Section 3 is divided into two parts: Sect. 3.1 examines the in situ differential flux of the energetic electrons and its impact on the D-region ionosphere for three different precipitation cases; Sect. 3.2 contains the comparison of the derived SEE rates with the other MSP-related charging rates for the different precipitation cases and at the altitudes 70 and $90 \mathrm{~km}$. Finally, in Sect. 4 we draw conclusions.

\section{Secondary electron emission from particles}

This section describes the fundamentals of secondary electron emission (SEE) from particles as it is widely used within the dusty plasma physics community (e.g., Meyer-Vernet, 1982). The SEE yield, i.e., the number of secondary electrons generated by one incoming electron, is a crucial parameter within the derivation of the SEE electron flux leaving particles which are subject to high-energy electron bombardement. The formalism of the SEE yield for nanometer-sized particles used in this study has been derived by Chow et al. (1993). In the following, we want to recapitulate the most important formula which has been used to derive the flux of secondary electrons generating from MSPs in the Earth's polar atmosphere where auroral electron precipitation is present.

The SEE yield was experimentally studied in the beginning of the last century but only for solid materials (e.g., Austin and Starke, 1902; Bruining, 1954). Chow et al. (1993) were the first to develop a SEE yield formalism for particles, which not only shows high yields for smaller particles but also reproduces the bulk SEE yield when applying larger particle radii $(r>1 \mu \mathrm{m})$. In Fig. 1 the basic principle of the SEE process within particles is described. Here, a primary electron enters a particle of radius $D / 2$ and penetrates a depth $x$ from the surface. On its way to the depth $x$ secondary electrons can be stimulated by the deposited energy of the primary electron. This secondary electron travels through the particle and is emitted in a direction under an angle $\phi$ with respect to the path of the primary electron. The distance a secondary electron has to travel to reach the surface of the particle is $l(\phi, x)$. This distance can be derived by applying the law of cosine and sine to the triangle in Fig. 1.

$$
\begin{aligned}
l(x, \phi)= & \left\{\left(\frac{D}{2}\right)^{2}+\left(\frac{D}{2}-x\right)^{2}-2\left(\frac{D}{2}\right)\left(\frac{D}{2}-x\right)\right. \\
& \left.\cdot \cos \left(\phi-\sin ^{-1}\left[\left(\frac{2}{D}\right)\left(\frac{D}{2}-x\right) \sin (\phi)\right]\right)\right\}
\end{aligned}
$$

The secondary yield $\delta$ for a primary electron with energy $E_{\mathrm{P}}$ is now the double integral covering the primary energy deposition and its use for secondary electron emission, as

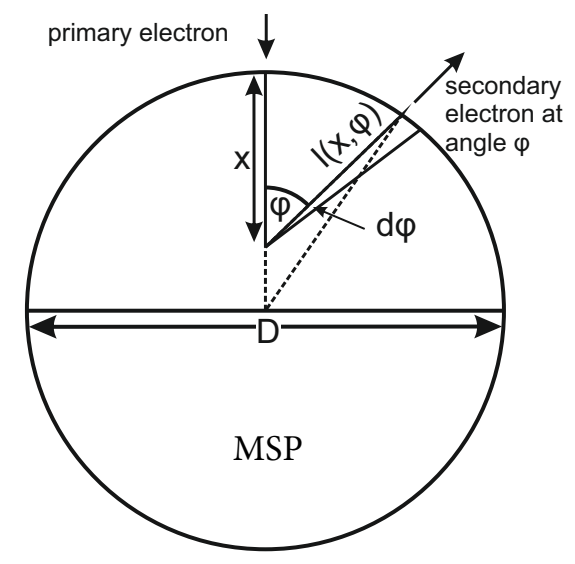

Figure 1. Principle of secondary electron emission in particles after Chow et al. (1993) (for description see text).

well as the absorption of secondaries traveling within the particle to its surface. The integration is carried out over the penetration depth $x$ and the angle $\phi$.

$$
\begin{aligned}
\delta\left(E_{\mathrm{P}}\right)= & \frac{1}{2} \int_{0}^{\min \left[D, x_{\max }\right]} K a\left[\left(E_{\mathrm{P}}\right)^{2}-a x\right]^{-1 / 2} \\
& \int_{0}^{\pi} \frac{1}{2} \sin (\phi) e^{-\alpha l(x, \phi)} \mathrm{d} \phi \mathrm{d} x
\end{aligned}
$$

Here, a is the Whiddington constant $\left(1.0 \times 10^{14} \mathrm{eV}^{2} \mathrm{~m}^{-1}\right.$ for metal particles, $0.92 \times 10^{14} \mathrm{eV}^{2} \mathrm{~m}^{-1}$ for insulator particles), $K$ is the efficiency of using primary electron energy to excite secondary electrons $(0.01$ for metal particles, 0.04 for insulator particles) and $\alpha$ is the inverse absorption length of secondary electrons $\left(1.0 \times 10^{8} 1 \mathrm{~m}^{-1}\right.$ within metal particles, $0.93 \times 10^{8} 1 \mathrm{~m}^{-1}$ within insulator particles). The numerical values for these parameters originate from calculations of Chow et al. (1993), which are based on experimental data from Bruining (1954) (metals) and Kanaya et al. (1978) (isolators). The integration over the penetration depth $x$ is carried out up to the minimum of either the maximum penetration depth $x_{\max }=\frac{E_{\mathrm{P}}^{2}}{a}$ or the particle diameter $D$. In the case of small primary electron energies, the electron is fully stopped within the particle and the integration is carried out up to $x_{\max }$. Though primary electrons with high energies can pass through the particle, in this case the integration limit is the particle diameter $D$.

Figure 2 shows the secondary electron yield as a function of primary electron energy for insulator and metal material. In this study we have used the yield for insulator particles, as MSPs most likely consist of insulator material (e.g., Rapp et al., 2012). The yield shows maximum values up of to 20 at characteristic energy levels. These energy values correspond to maximum penetration depths in the region of the particle diameter. At lower energies the primary electron is 


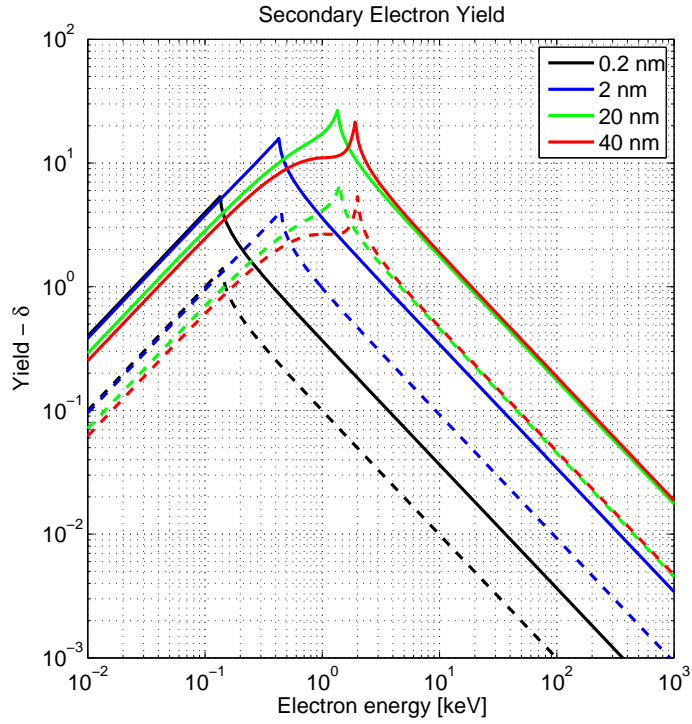

Figure 2. Secondary electron yield as a function of primary electron energy for metallic (dashed lines) and isolator (solid lines) particles of different sizes.

still stopped within the particle and the yield still shows relatively high values. Above these characteristic energies the secondary yield decays exponentially, as the primary electrons deposit only a small fraction of their energy into the excitation of secondary electrons.

The number of secondary electrons leaving a particle of radius $r$ per second depends on the charge of the particle. If the particle carries a negative charge of $Z \cdot e$, the flux of secondary electrons $J_{\mathrm{sec}}$ is given by the following (MeyerVernet, 1982):

$J_{\mathrm{sec}}=\pi r^{2} \int_{-e \Phi}^{\infty} \frac{\partial j_{e}}{\partial E}\left(1+\frac{e \Phi}{E}\right) \delta(E) \mathrm{d} E$.

Here, the integration is carried out over the energy range of the differential flux of primary electrons $\frac{\partial j_{e}}{\partial E}$ and the secondary yield $\delta . \Phi$ is the surface potential of the particle of radius $r$ and with charge number $Z$. The lower limit of the integration is the kinetic energy a primary electron needs to reach the surface of the negatively charged particle.

In the case of positively charged particles, the emitted secondary electrons have to overcome an attractive Coulomb force and the above equation $J_{\text {sec }}$ changes as follows:

$$
\begin{aligned}
J_{\mathrm{sec}}= & e^{-\Phi / k_{\mathrm{B}} T_{s}}\left(1+\frac{\Phi}{k_{\mathrm{B}} T_{s}}\right) \pi r^{2} \\
& \int_{0}^{\infty} \frac{\partial j_{e}}{\partial E}\left(1+\frac{e \Phi}{E}\right) \delta(E) \mathrm{d} E .
\end{aligned}
$$

Here, $k_{\mathrm{B}} T_{s}$ is the kinetic energy of a secondary electron after leaving a particle. The velocity distribution of the secondaries is Maxvellian, and they are in the range of 1 to $5 \mathrm{eV}$
(Goertz, 1989); we have arbitrarily used $3 \mathrm{eV}$ in this study. This value only has an impact on the SEE rate of multiple positively charged particles.

In the following, the SEE formalism for particles is applied to MSPs. These MSPs are not only subject to SEE induced by polar electron precipitation but are also charged within the D-region ionosphere by processes like electron and ion attachment as well as photodetachment and photoionization. The process of ion attachment to MSPs depicts a generalization of different processes on the atomic scale depending on the ion type, i.e., electron transfer, proton transfer, cation attachment and anion attachment. These processes are approached classically by, e.g., Natanson (1960), Fuchs (1963) and Hoppel and Frick (1986) and are generalized into one reaction rate coefficient dependent on temperature, ion mass and charge and MSP charge state (Rapp, 2000).

\section{SEE application to MSPs within the polar ionosphere}

Secondary electron emission is only relevant at polar latitudes. Here, the Earth's magnetic field lines penetrate into the atmosphere and allow energetic electrons from the magnetosphere to enter. These high-energy electrons contribute significantly to the ionization in the lower ionosphere (e.g., Frahm et al., 1997).

In order to make reliable statements on the importance of SEE for the charge state of MSPs within the D region, we model the polar ionosphere with the Sodankylä Ion and Neutral Chemistry (SIC) model (Verronen et al., 2005; Turunen et al., 2009) and compare standard MSP-related charging processes with SEE. The model derives the concentrations of 44 positive ion species, 28 negative ion species and 35 neutral species from 20 to $150 \mathrm{~km}$. The SIC model enables the implementation of different electron precipitation spectra as a source of ionization additional to solar UV and EUV radiation. This study uses the SIC model version with MSPs included into the full ion reaction scheme as recently described in Baumann et al. (2015).

The following analysis is carried out at 90 and $70 \mathrm{~km}$ altitude. We have chosen these altitudes for investigating the effectiveness of SEE on the charging of MSPs, because they represent two different states of the lower ionosphere. At $90 \mathrm{~km}$ the ionosphere is governed by the presence of free electrons and positive ions only. At $70 \mathrm{~km}$, negatively charged ions can exist in addition to free electrons as another type of negative charge carriers. In addition, the size distributions of MSPs are different at both altitudes. Just after their formation at $90 \mathrm{~km}$ altitude, the radii of MSPs are rather small $(<1 \mathrm{~nm})$. During sedimentation down to $70 \mathrm{~km}$, MSPs grow through coagulation to radii $>1 \mathrm{~nm}$ (e.g., Megner et al., 2006). 


\subsection{Electron precipitation and D-region conditions}

Electron precipitation is often described by the differential flux of electrons $\partial j_{e} / \partial E$ entering the atmosphere from the magnetosphere. This quantity can be derived from measurements of incoherent-scatter radars and by instruments on board satellites and sounding rockets (e.g., Rees, 1969; Miyoshi et al., 2015, and references therein) and is usually given for the top of the atmosphere. While penetrating the atmosphere, energetic electrons collide with atmospheric molecules; they lose energy and can get absorbed completely. The altitude of complete absorption of the energetic electron depends on electron energy and the density of the atmosphere. Fang et al. (2010) derived a normalized energy deposition $\mathrm{f}$ to describe the altitude region where electrons with a certain energy are absorbed. We have adapted this formalism to derive in situ energetic electron spectra for 70 and $90 \mathrm{~km}$. These spectra are then used in Eqs. (3) and (4) to derive the secondary electron flux $J_{\text {sec }}$.

In the left panel of Fig. 3 the spectra for three different cases of electron precipitation are plotted (different line shapes), namely at the top of the atmosphere, at $90 \mathrm{~km}$ altitude and $70 \mathrm{~km}$ altitude (different colors). The three cases are defined as follows: the "weak" electron precipitation case is the spectrum measured during a pulsating aurora event over Troms $\varnothing$ (Miyoshi et al., 2015). The "medium" precipitation case corresponds to the "Hard" spectra shown in Osepian and Kirkwood (1996). The electron precipitation of the "strong" case is the medium precipitation case increased by a factor of 100 ; i.e., this is an unrealistically strong precipitation used as a test case. The medium and strong cases are Maxwellian spectra of the form

$\frac{\partial j_{e}}{\partial E}(E)=\frac{Q_{0}}{2 E_{0}^{3}} \exp \left(\frac{-E}{E_{0}}\right)$.

Here $Q_{0}$ is the total energy flux of the precipitating electrons and $E_{0}$ is the characteristic energy of the spectrum.

In order to get a complete view of the energetic electrons in the Earth's atmosphere, we also consider the fraction of secondary electrons emitted in situ from the atmospheric species oxygen and nitrogen. As the primary electrons are absorbed within the atmosphere, their kinetic energy is degraded through elastic collision with atmospheric molecules, leading to the emission of atmospheric secondary electrons (Rees et al., 1969). According to laboratory measurements of Opal et al. (1971), the energy distribution of secondary electrons from $\mathrm{O}_{2}$ and $\mathrm{N}_{2}$ does not depend on the energy of the primary electrons and is within the sub-kiloelectronvolt energy range. To study the "tertiary" electron emission (TEE) from MSPs, we have used an atmospheric secondary differential electron flux measured at $105 \mathrm{~km}$ altitude under auroral conditions (see Fig. 3, data collected by Doering, published within Fig. 4 of Pfister, 1967). Pfister (1967) also shows similar atmospheric secondary differential fluxes for higher altitudes. We assume that this atmospheric secondary electron
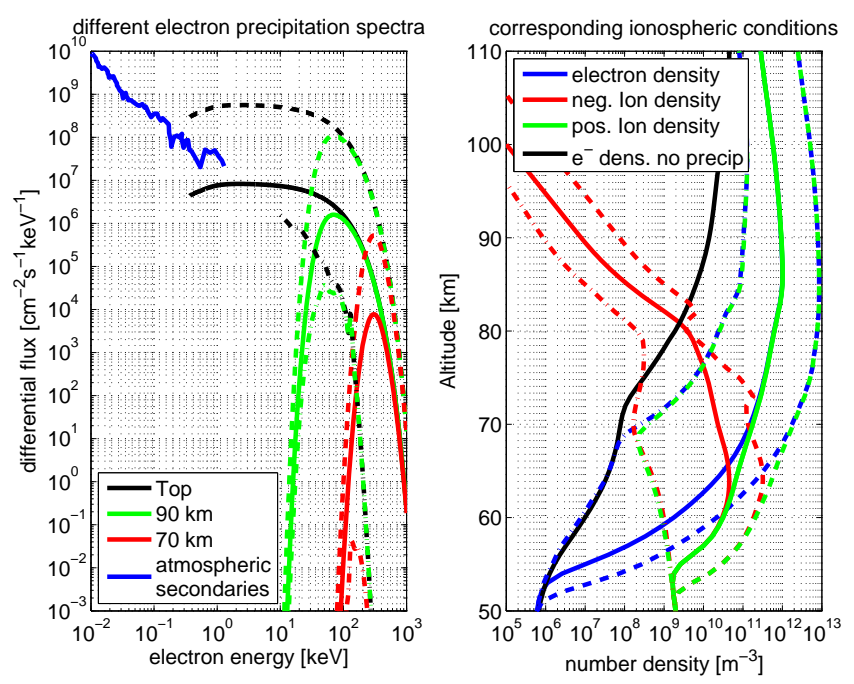

Figure 3. (Left) Electron precipitation spectra for weak (dashdotted, data published in Miyoshi et al., 2015), medium (solid) and strong (dashed) conditions; spectra at the top of the atmosphere (black) and remnants at $90 \mathrm{~km}$ (green) and $70 \mathrm{~km}$ (red); and atmospheric secondary electron spectrum measured at $105 \mathrm{~km}$ by Doering (data taken from Fig. 4. of Pfister, 1967). (Right) corresponding to the precipitation cases the electron density (blue), negative ion density (red) and positive ion density (green) have been modeled using the SIC model together with the electron density (black) for a model run without electron precipitation.

differential flux is typical at auroral latitudes and also applies to lower altitudes as long as primary electrons are absorbed within these altitudes. The causal primary electron flux for the atmospheric secondary flux shown in Fig. 3 is only comparable to the differential flux measured by Miyoshi et al. (2015) within a pulsating aurora (cf. Fig. 2 of Pfister, 1967). This atmospheric secondary flux cannot be used for the other precipitation cases as it depends on the strength of the initial electron precipitation (Rees, 1969). To the authors' knowledge, there are no atmospheric secondary electron spectra available whose initial electron precipitation corresponds to the other precipitation cases used in this study.

These three different precipitation cases have been used within the SIC model to determine the state of the lower ionosphere. The aim of this calculation is to put the secondary electron emission into a context of MSP-related charging processes within the $\mathrm{D}$-region ionosphere. In the right panel of Fig. 3 the results of these model runs are shown. Here the electron, positive and negative ion densities are plotted in the corresponding line shape of the different precipitation cases of the left panel. The positive and negative ion number density is the the sum of the number densities of the various ion species derived by SIC. In addition, there we also show one electron density profile for comparison, which has been modeled for quiet ionospheric conditions, i.e., in the absence of electron precipitation. 


\subsection{Comparison of MSP-related charging rates and discussion}

The abovementioned three electron precipitation cases are compared to each other, in order to identify whether SEE is an important charging process for MSPs. This comparison includes the discussion of the SEE rates and other MSPrelated charging rates, i.e., plasma attachment and charging by solar photons. To derive plasma attachment rates which are equivalent to the SEE rate, the electron and ion number densities modeled with the SIC model are multiplied with the attachment reaction rate coefficients (Natanson, 1960; Rapp, 2000). In addition to the SEE and plasma attachment we also consider photodetachment and photoionization of MSPs in this study. According to Rapp (2009) we assumed $\mathrm{Fe}_{2} \mathrm{O}_{3}$ (Hematite) to be a plausible MSP analogue and derived the detachment and ionization rates for solar radiation at a solar zenith angle of $66^{\circ}$ at the location Troms $\varnothing\left(69^{\circ} \mathrm{N}\right)$. Nevertheless, research on the material properties of MSPs is still ongoing and is needed to further constrain the optical characteristics of MSPs.

In the panels of Fig. 4 the rates for different MSP sizes are plotted for the processes of plasma attachment to neutral MSPs, photodetachment of negatively charged MSPs, photoionization of neutral MSPs and the secondary electron emission from neutral MSPs induced by primary electrons (secondary) and atmospheric secondary electrons (tertiary). As discussed earlier, the rates for these processes are derived on the basis of three different precipitation cases and the corresponding ionospheric modeling. The process rates have been derived for the altitudes 90 and $70 \mathrm{~km}$.

For the case of SEE induced by primary electron precipitation the energy of the energetic electrons is so high that they pass through the MSP and deposit only a small fraction of their energy into the MSP; here the SEE yields are far below one (compare Fig. 2). Moreover, the SEE rate from MSPs shows in general a size dependency. The huge difference of several orders of magnitude of the SEE rate between the smallest $(0.2 \mathrm{~nm})$ and biggest particles $(40 \mathrm{~nm})$ has two main reasons. Firstly, the SEE yield is a function of particle size for high primary electron energies; namely it is more then one order of magnitude smaller for $0.2 \mathrm{~nm}$ MSPs then for $40 \mathrm{~nm}$ particles.

Secondly, the SEE rate grows with the square of the MSP radii (compare Eq. 3).

Concerning electron precipitation, the absorption of energetic electrons is already strong down to $90 \mathrm{~km}$; only electrons with energies higher than $10 \mathrm{keV}$ can reach this altitude. For $70 \mathrm{~km}$ altitude this value is $100 \mathrm{keV}$. This absorption limits the available number of energetic electrons for SEE from MSPs, resulting in significantly lower SEE rates at $70 \mathrm{~km}$ compared to $90 \mathrm{~km}$.

There is also absorption of solar photons within the Earth's atmosphere that leads to different solar spectra available for photodetachment and photoionization of MSPs at 70 and
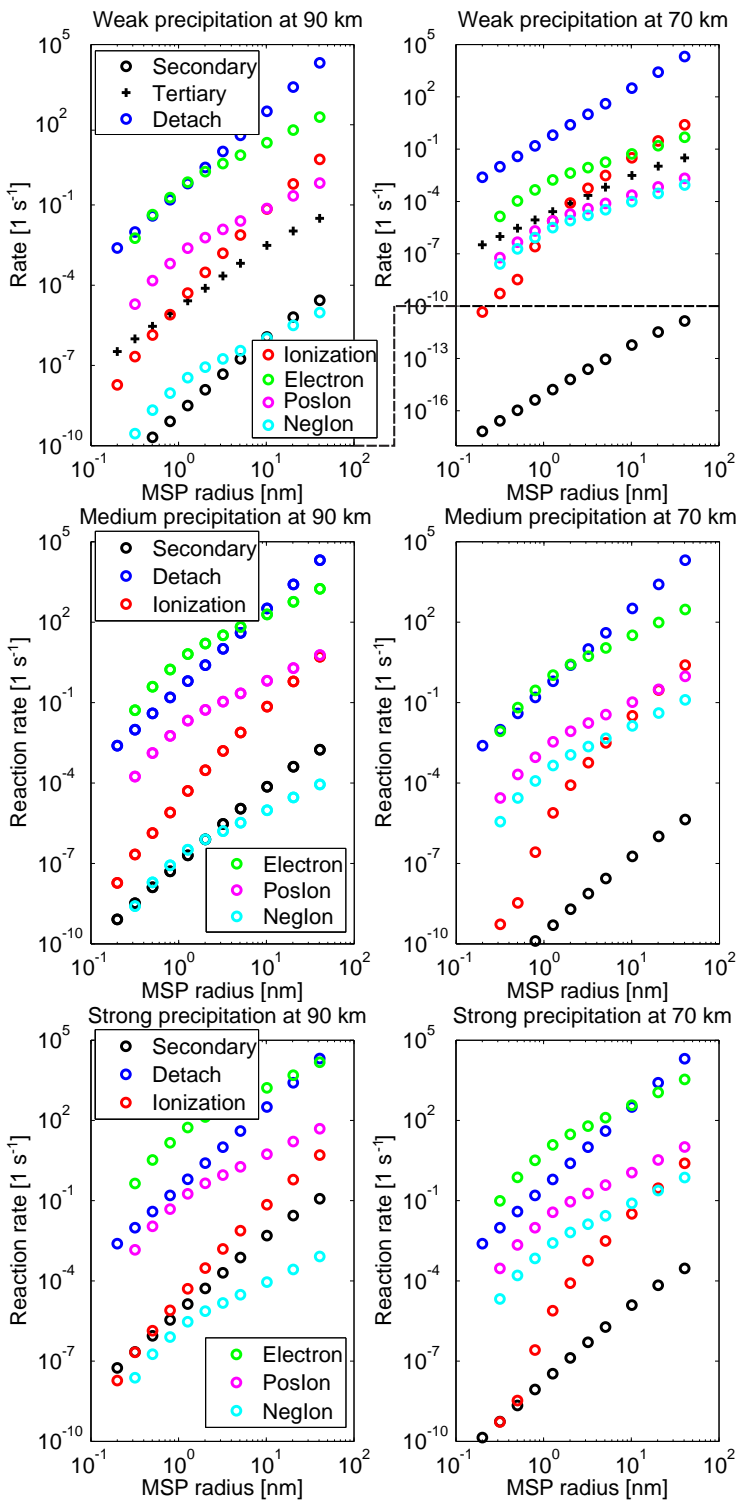

Figure 4. Charging rates of different particle sizes corresponding to the weak (top), medium (middle) and strong (bottom) electron precipitation case for secondary electron emission (black); particle ionization (red); electron detachment from negatively charged particles (blue; and electron (green), negative (cyan) and positive ion attachment (magenta) for ionospheric conditions at $90 \mathrm{~km}$ (left) and $70 \mathrm{~km}$ (right) altitude.

$90 \mathrm{~km}$. This UV photon absorption leads to a reduction of the photoionization rates at $70 \mathrm{~km}$ by a factor of $3 \mathrm{com}$ pared to $90 \mathrm{~km}$. For the photodetachment this reduction is even smaller, since also photons of lower energies within the visible spectrum can detach electrons from negatively charged MSPs. These photons are not at all absorbed by the atmosphere, and as a consequence the photodetachment rate shows only a small reduction at the second decimal place, i.e., due to UV absorption (e.g., Rapp, 2009). The photode- 
tachment and photoionization of MSPs do not change for different electron precipitation cases.

The attachment rate of ions and electrons to neutral MSPs varies with every precipitation case and altitude; i.e., the rate is given by the product of reaction rate coefficient and plasma number density (see Fig. 3 right panel). We have decided to show only the neutral case of the possible attachment of plasma to neutral MSPs. The reaction rate coefficients for the repulsive case of plasma attachment to identically charged particles are many orders of magnitude smaller compared to the reaction rate coefficients for the charging of neutral MSPs and hence can be neglected. Moreover, the reaction rate coefficient of the attractive case of plasma attachment to MSPs is of the same order of magnitude as the neutral case (Rapp, 2000). Showing these in Fig. 4 does not provide additional information.

The general comparison of the process rates concerning charging of MSPs in Fig. 4 shows that in all studied precipitation cases and at both altitudes the rates of the electron attachment to neutral MSPs and the photodetachment of negatively charged MSPs are the governing processes for the charge state of MSPs. The process rates of secondary electron emission, neither induced by primary electrons (i.e., SEE) nor induced by atmospheric secondary electrons (i.e., TEE), are several orders of magnitude below these attachment and detachment processes.

A detailed comparison at $90 \mathrm{~km}$ for the weak and medium precipitation cases shows that the SEE rate from neutral MSPs is of the same magnitude as the attachment rate of negative ions to MSPs. In the strong precipitation case the SEE rate even overcomes the negative ion attachment rate to MSPs. As the additional ionization by electron precipitation grows from the weak case to the strong case, the additional free electrons lead to the production of more negative ions even above $80 \mathrm{~km}$, where the presence of atomic oxygen usually destroys negatively charged ions effectively. At the same time the SEE rate grows as the available amount of energetic electrons present at $90 \mathrm{~km}$ increases with the strength of the electron precipitation. In the strong precipitation case the SEE rate reaches nearly the MSP photoionization process rate. But the SEE rate at $90 \mathrm{~km}$ is several orders of magnitude lower than the attachment of positive ions and electrons to MSPs as well as the very effective photodetachment of negatively charged particles in all three precipitation cases.

At $70 \mathrm{~km}$ the SEE rate induced by primary electrons is even smaller as there are fewer available energetic electrons. In the weak and medium precipitation case the SEE rate lies far below all other process rates. Even the attachment of negative ions is much greater, since the number density of negative ions at $70 \mathrm{~km}$ is much higher. The strong precipitation case shows SEE rates for small MSPs as high as the photoionization rates, but these values are not really relevant as at $70 \mathrm{~km}$ altitude these small MSPs exist only sparsely (e.g., Megner et al., 2006). For greater MSP sizes SEE does not reach any other MSP charging rate either in the weak and medium case or in the strong precipitation case.

The question now is how often SEE from MSPs occurs in the the polar ionosphere. We have studied three precipitation cases, of which the weak and medium cases happen in the polar atmosphere and the strong case has more the nature of a thought experiment with extraordinarily severe electron precipitation. The electron precipitation for pulsating aurora (weak case), caused by energetic electrons with tens of kiloelectronvolts of energy (Miyoshi et al., 2015), occurs frequently during minor geomagnetic activity. Furthermore, electrons trapped within the Earth's magnetosphere can be accelerated to relativistic energies (e.g., Reeves et al., 2013), which can be injected into the Earth's atmosphere during geomagnetic storms; this scenario is comparable to our medium precipitation case. This has the following consequences for the occurrence of the effect of SEE during different electron precipitation cases: the weak precipitation case causes, despite the relatively frequent occurrence, a SEE effect on MSPs that is marginal compared to other MSP-related charging processes. The medium precipitation case occurs sparsely during 1 year, and the effect of SEE on the charge of MSPs is still small. Even an unrealistic increase of the electron precipitation results in SEE rates which are still not significant for the charge state of MSPs.

The consideration to study the TEE process originated from two facts. Firstly, the secondary electron yield at energetic electron energies below $1 \mathrm{keV}$ is about one order of magnitude higher than the yield at energies above $10 \mathrm{keV}$ (cf. Fig. 2). Secondly, the atmospheric secondary differential flux is also much higher than the available primary electron flux at 90 and $70 \mathrm{~km}$. It turns out that the TEE rate is higher than the SEE rate but still two orders of magnitude lower than the electron attachment to MSPs and photodetachment of negatively charged MSPs. The derived TEE rate is only valid within the weak precipitation case, because the used atmospheric secondary flux corresponds to a primary electron flux that is comparable to the weak precipitation case. Therefore, the TEE rate is only plotted in the weak precipitation panels of Fig. 4. The tertiary electron emission rate shows a smaller size dependence, this is because within the subkiloelectronvolts electron energy range the secondary yield is independent of the MSP sizes. This is because the electrons deposit all their kinetic energy into the MSPs within this energy range. At the altitude of $90 \mathrm{~km}$, the TEE rate is only comparable to the photoionization rate and exceeds the negative ion attachment rate but cannot reach the positive ion attachment rate. At $70 \mathrm{~km}$, the TEE rate is of the same order of magnitude as the positive and negative ion attachment rates as well as the photoionization rate.

It has to be noted that the nature of the tertiary electron emission is unsettled at the moment. In particular, the differential flux of atmospheric secondary electrons at altitudes below $100 \mathrm{~km}$ and for different kinds of electron precipitation strengths is not known. In addition, we assume for TEE from 
MSPs that the atmospheric secondary electrons have to have at least $10 \mathrm{eV}$ of kinetic energy in order to enter a MSP and excite an electron that has enough energy to leave the particle. Therefore, our quantification of the TEE rate can be seen as a rough estimate only. Furthermore, we think that MSP charging by TEE cannot be of the same importance as electron attachment to MSPs. The existence of an atmospheric secondary electron flux more than three orders of magnitude higher than used in this study, in order to come up with TEE rates comparable to the electron attachment rates, is not plausible.

\section{Conclusions}

Energetic particle precipitation is a common phenomenon occurring within the polar latitudes and especially affects the lower ionosphere. As shown in Fig. 4, electron precipitation enhances the number density of plasma components by several orders of magnitude. In this study we examined secondary electron emission from meteoric smoke particles. MSPs are produced and exist within the same altitude region of 70 to $110 \mathrm{~km}$, where precipitating electrons deposit a substantial amount of their energy. Here, these MSPs can be subject to secondary electron emission induced by this energetic particle precipitation (SEE). We have studied the effect of SEE from MSPs within the polar ionosphere and compared this charging process with other MSP-related charging mechanisms in the D-region and lower E-region ionosphere. To derive realistic SEE rates, we have used in situ energetic electron spectra and a SEE yield for particles (Chow et al., 1993). The ionosphere has been characterized by the SIC model including the weak, medium and strong electron precipitation cases which have been studied in more detail.

It is also possible that atmospheric secondary electrons from gaseous components can cause tertiary electron emission from MSPs. The TEE rates have been derived from an atmospheric secondary electron differential flux measured at $105 \mathrm{~km}$ altitude (Pfister, 1967) using the same yield formalism. However, the robustness of the TEE results is rather weak, as atmospheric secondary electron spectra at lower altitudes and the corresponding primary electron spectra are not available. Additional in situ and laboratory experiments are needed for a solid quantification of the TEE effect.

Concerning the charge state of MSPs, the processes SEE and TEE do not play a significant role in all three considered precipitation cases at the studied altitudes of 70 and $90 \mathrm{~km}$. In general, the direct response of the polar ionosphere on electron precipitation, i.e., an enhancement of the number density of plasma components due to additional ionization in the ionosphere, is the main driver for the charge state of MSPs. The charging of MSPs through electron attachment and decharging through photodetachment of negatively charged MSPs are the relevant processes.
Nevertheless, there might be an influence of SEE and TEE from MSPs through the production of additional electrons in the lower ionosphere. That could be of interest to explain unresolved phenomena within the polar atmosphere during energetic electron precipitation.

Acknowledgements. The authors thank P. T. Verronen for valuable discussions on the topic.

The article processing charges for this open-access publication were covered by a Research

Centre of the Helmholtz Association.

The topical editor, A. J. Kavanagh, thanks the one anonymous referee for help in evaluating this paper.

\section{References}

Abbas, M. M., Tankosic, D., LeClair, A. C., and Spann, J. F.: Charging of Dust Grains in Astrophysical Environments by Secondary Electron Emissions, Astrophys. J., 756, 41, doi:10.1088/0004637X/756/1/41, 2012.

Anderson, P. and Koons, H.: Spacecraft charging anomaly on a lowaltitude satellite in an aurora, J. Spacecraft Rockets, 33, 734-738, doi:10.2514/3.26828, 1996.

Asmus, H., Robertson, S., Dickson, S., Friedrich, M., and Megner, L.: Charge balance for the mesosphere with meteoric dust particles, J. Atmos. Sol.-Terr. Phy., 127, 137-149, doi:10.1016/j.jastp.2014.07.010, 2015.

Austin, L. and Starke, H.: Ueber die Reflexion der Kathodenstrahlen und eine damit verbundene neue Erscheinung secundärer Emission, Ann. Phys.-Berlin, 314, 271-292, doi:10.1002/andp.19023141003, 1902.

Balcon, N., Payan, D., Belhaj, M., Tondu, T., and Inguimbert, V.: Secondary electron emission on space materials: Evaluation of the total secondary electron yield from surface potential measurements, Plasma Science, IEEE T. Plasma Sci., 40, 282-290, doi:10.1109/TPS.2011.2172636, 2012.

Baumann, C., Rapp, M., Kero, A., and Enell, C.-F.: Meteor smoke influences on the D-region charge balance - review of recent in situ measurements and one-dimensional model results, Ann. Geophys., 31, 2049-2062, doi:10.5194/angeo-31-20492013, 2013.

Baumann, C., Rapp, M., Anttila, M., Kero, A., and Verronen, P. T.: Effects of meteoric smoke particles on the $\mathrm{D}$ region ion chemistry, J. Geophys. Res.-Space, 120, 10823-10839, doi:10.1002/2015JA021927, 2015.

Bruining, H.: Physics and Applications of Secondary Electron Emission, Pergamon Press Ltd., Maxwell House, edited by: Fry, D. W., ISBN: 0080090141, 1954.

Chow, V. W., Mendis, D. A., and Rosenberg, M.: Role of grain size and particle velocity distribution in secondary electron emission in space plasmas, J. Geophys. Res.-Space, 98, 19065-19076, doi:10.1029/93JA02014, 1993.

Fang, X., Randall, C. E., Lummerzheim, D., Wang, W., Lu, G., Solomon, S. C., and Frahm, R. A.: Parameterization of mo- 
noenergetic electron impact ionization, Geophys. Res. Lett., 37, L22106, doi:10.1029/2010GL045406, 2010.

Frahm, R. A., Winningham, J. D., Sharber, J. R., Link, R., Crowley, G., Gaines, E. E., Chenette, D. L., Anderson, B. J., and Potemra, T. A.: The diffuse aurora: A significant source of ionization in the middle atmosphere, J. Geophys. Res.-Atmos, 102, 28203-28214, doi:10.1029/97JD02430, 1997.

Friedrich, M., Rapp, M., Blix, T., Hoppe, U.-P., Torkar, K., Robertson, S., Dickson, S., and Lynch, K.: Electron loss and meteoric dust in the mesosphere, Ann. Geophys., 30, 1495-1501, doi:10.5194/angeo-30-1495-2012, 2012.

Fuchs, N.: On the stationary charge distribution on aerosol particles in a bipolar ionic atmosphere, Geofisica pura e applicata, 56, 185-193, doi:10.1007/BF01993343, 1963.

Goertz, C. K.: Dusty Plasmas in the Solar System, Rev. Geophys., 27, 271-292, doi:10.1029/RG027i002p00271, 1989.

Havnes, O., Trøim, J., Blix, T., Mortensen, W., Næsheim, L., Thrane, E., and Tønnesen, T.: First detection of charged dust particles in the Earth's mesosphere, J. Geophys. Res., 101, 1083910847, doi:10.1029/96JA00003, 1996.

Hervig, M. E., Gordley, L. L., Deaver, L. E., Siskind, D. E., Stevens, M. H., Russell, J. M., Bailey, S. M., Megner, L., and Bardeen, C. G.: First Satellite Observations of Meteoric Smoke in the Middle Atmosphere, Geophys. Res. Lett., 36, L18805, doi:10.1029/2009GL039737, 2009.

Hoppel, W. A. and Frick, G. M.: Ion-Aerosol Attachment Coefficients and the Steady-State Charge Distribution on Aerosols in a Bipolar Ion Environment, Aerosol Sci. Tech., 5, 1-21, doi:10.1080/02786828608959073, 1986.

Kanaya, K., Ono, S., and Ishigaki, F.: Secondary electron emission from insulators, J. Phys. D Appl. Phys., 11, 2425, doi:10.1088/0022-3727/11/17/015, 1978.

Katz, I., Mandell, M., Jongeward, G., and Gussenhoven, M. S.: The importance of accurate secondary electron yields in modeling spacecraft charging, J. Geophys. Res.-Space, 91, 13739-13744, doi:10.1029/JA091iA12p13739, 1986.

Megner, L., Rapp, M., and Gumbel, J.: Distribution of meteoric smoke - sensitivity to microphysical properties and atmospheric conditions, Atmos. Chem. Phys., 6, 4415-4426, doi:10.5194/acp-6-4415-2006, 2006.

Meyer-Vernet, N.: Flip-flop of electric potential of dust grains in space, Astron. Astrophys., 105, 98-106, 1982.

Miyoshi, Y., Oyama, S., Saito, S., Kurita, S., Fujiwara, H., Kataoka, R., Ebihara, Y., Kletzing, C., Reeves, G., Santolik, O., Clilverd, M., Rodger, C. J., Turunen, E., and Tsuchiya, F.: Energetic electron precipitation associated with pulsating aurora: EISCAT and Van Allen Probe observations, J. Geophys. Res.-Space, 120, 2754-2766, doi:10.1002/2014JA020690, 2015.

Natanson, G. L.: On the theory of the charging of atmospheric aerosol particles as a result of capture of gas ions, Sov. Phys. Tech. Phys., 5, 538-551, 1960.

Opal, C. B., Peterson, W. K., and Beaty, E. C.: Measurements of Secondary Electron Spectra Produced by Electron Impact Ionization of a Number of Simple Gases, J. Chem. Phys., 55, 41004106, doi:10.1063/1.1676707, 1971.

Osepian, A. and Kirkwood, S.: High-energy electron fluxes derived from EISCAT electron density profiles, J. Atmos. Terr. Phy., 58, 479-487, doi:10.1016/0021-9169(95)00050-X, 1996.
Pfister, W.: Auroral Invesitgations by Means of Rockets, Space Sci. Rev., 7, 642-688, doi:10.1007/BF00542893, 1967.

Plane, J. M., Saunders, R. W., Hedin, J., Stegman, J., Khaplanov, M., Gumbel, J., Lynch, K. A., Bracikowski, P. J., Gelinas, L. J., Friedrich, M., Blindheim, S., Gausa, M., and Williams, B. P.: A combined rocket-borne and ground-based study of the sodium layer and charged dust in the upper mesosphere, J. Atmos. Sol.Terr. Phy., 118, 151-160, doi:10.1016/j.jastp.2013.11.008, 2014.

Rapp, M.: Capture rates of electrons and positive ions by mesospheric aerosol particles, J. Aerosol Sci., 31, 1367-1369, doi:10.1016/S0021-8502(00)00030-6, 2000.

Rapp, M.: Charging of mesospheric aerosol particles: the role of photodetachment and photoionization from meteoric smoke and ice particles, Ann. Geophys., 27, 2417-2422, doi:10.5194/angeo-27-2417-2009, 2009.

Rapp, M., Plane, J. M. C., Strelnikov, B., Stober, G., Ernst, S., Hedin, J., Friedrich, M., and Hoppe, U.-P.: In situ observations of meteor smoke particles (MSP) during the Geminids 2010: constraints on MSP size, work function and composition, Ann. Geophys., 30, 1661-1673, doi:10.5194/angeo-30-1661-2012, 2012.

Rees, M.: Auroral electrons, Space Sci. Rev., 10, 413-441, doi:10.1007/BF00203621, 1969.

Rees, M., Stewart, A., and Walker, J.: Secondary electrons in aurora, Planet. Space Sci., 17, 1997-2008, doi:10.1016/00320633(69)90137-8, 1969.

Reeves, G. D., Spence, H. E., Henderson, M. G., Morley, S. K., Friedel, R. H. W., Funsten, H. O., Baker, D. N., Kanekal, S. G., Blake, J. B., Fennell, J. F., Claudepierre, S. G., Thorne, R. M., Turner, D. L., Kletzing, C. A., Kurth, W. S., Larsen, B. A., and Niehof, J. T.: Electron Acceleration in the Heart of the Van Allen Radiation Belts, Science, 341, 991-994, doi:10.1126/science.1237743, 2013.

Rosinski, J. and Snow, R. H.: Secondary particulate matter from meteor vapors, J. Meteorol., 18, 736-745, 1961.

Strelnikova, I., Rapp, M., Raizada, S., and Sulzer, M.: Meteor smoke particle properties derived from Arecibo incoherent scatter radar observations, Geophys. Res. Lett., 34, L15815, doi:10.1029/2007GL030635, 2007.

Turunen, E., Verronen, P. T., Seppälä, A., Rodger, C. J., Clilverd, M. A., Tamminen, J., Enell, C.-F., and Ulich, T.: Impact of different energies of precipitating particles on NOx generation in the middle and upper atmosphere during geomagnetic storms, J. Atmos. Sol.-Terr. Phy., 71, 1176-1189, doi:10.1016/j.jastp.2008.07.005, 2009.

Verronen, P. T., Seppälä, A., Clilverd, M. A., Rodger, C. J., Kyrölä, E., Enell, C.-F., Ulich, T., and Turunen, E.: Diurnal variation of ozone depletion during the October-November 2003 solar proton events, J. Geophys. Res.-Space, 110, A09S32, doi:10.1029/2004JA010932, 2005.

Walch, B., Horányi, M., and Robertson, S.: Charging of dust grains in plasma with energetic electrons, Phys. Rev. Lett., 75, 838, doi:10.1103/PhysRevLett.75.838, 1995.

Wilms, H., Rapp, M., and Kirsch, A.: Nucleation of mesospheric cloud particles: Sensitivities and limits, J. Geophys. Res.-Space, 121, 2621-2644, doi:10.1002/2015JA021764, 2016. 